\title{
A narrative review of ultrasound-guided serratus anterior plane block
}

\author{
Cuiyu Xie" ${ }^{1 \#}$, Guo $\operatorname{Ran}^{2 \#}$, Daoqi Chen ${ }^{3}$, Yao Lu' ${ }^{1}$ \\ ${ }^{1}$ Department of Anesthesiology, The First Affiliated Hospital of Anhui Medical University, Hefei, China; ${ }^{2}$ Department of Anesthesiology, \\ Eye \& ENT Hospital, Fudan University, Shanghai, China; ${ }^{3}$ Department of Anesthesiology, The First College of Clinical Medicine, Anhui Medical \\ University, Hefei, China \\ Contributions: (I) Conception and design: All authors; (II) Administrative support: None; (III) Provision of study materials or patients: C Xie, G Ran; \\ (IV) Collection and assembly of data: C Xie, G Ran; (V) Data analysis and interpretation: All authors; (VI) Manuscript writing: All authors; (VII) \\ Final approval of manuscript: All authors. \\ \#These authors contributed equally to this work. \\ Correspondence to: Yao Lu. Department of Anesthesiology, The First Affiliated Hospital of Anhui Medical University, No.218 Jixi Road, Hefei 230022, \\ China. Email: luyao@ahmu.edu.cn.
}

\begin{abstract}
Ultrasound-guided serratus anterior plane block (SAPB) is located using ultrasound at the level of the midaxillary line and the fifth rib, and a certain amount of local anesthetics is injected either superficially or deeply into the serratus anterior muscle, blocking the third to sixth intercostal nerves, the long thoracic and thoracodorsal nerves. It is mainly used in breast surgeries, rib fractures and thoracotomy to manage the pain of the anterolateral chest wall. The surgery of anterolateral chest wall is often accompanied by severe postoperative pain, leading to postoperative infection, atelectasis and other complications, and prolonged hospitalization. However, effective pain management can reduce the occurrence of postoperative pulmonary complications, promote patients to get out of bed as soon as possible, and accelerate the recovery of patients. Recently, with the development of ultrasonic technology and equipment, SAPB has entered the era of visualization, further improving the safety and success rate of operations. SAPB, as a new technology of regional block, has a higher positioning accuracy, a higher success rate, lesser complications, and simpler and more effective postoperative analgesia effect. Compared with thoracic epidural analgesia and thoracic paravertebral block, SAPB has a good ultrasonic anatomical basis; thus, has a broad application prospect in clinics. In this paper, the SAPB in clinical application was reviewed.
\end{abstract}

Keywords: Ultrasound; serratus anterior plane block (SAPB); postoperative analgesia; clinical application

Submitted Aug 04, 2020. Accepted for publication Dec 01, 2020.

doi: 10.21037/apm-20-1542

View this article at: http://dx.doi.org/10.21037/apm-20-1542

\section{Introduction}

Recently, with the development of ultrasonic technology, a higher success rate, lesser complications, and simpler and more effective postoperative analgesia effect are acquired through ultrasound. Ultrasound-guided serratus anterior plane block (SAPB) is located through ultrasound at the level of the midaxillary line and the fifth rib, and a certain amount of local anesthetics is injected either superficially or deeply into the serratus anterior muscle, blocking the third to sixth intercostal nerves, the long thoracic and thoracodorsal nerves. It has been used in breast surgeries, rib fractures, and thoracotomy to manage pain of the anterolateral chest wall. In this review, we briefly describe the anatomy, operation, drug selection, block range, and clinical application of SAPB. We present the following article in accordance with the Narrative Review reporting checklist (available at http://dx.doi.org/10.21037/apm20-1542). 


\section{Methods}

This review was reorganized upon the research of SAPB from 2013 to 2020 .

\section{Discussion}

\section{Anatomical basis and operation of SAPB}

The serratus anterior is a large flat muscle located on the wall of the rib cage. It starts from outside the upper eighth and ninth ribs and ends at the medial edge and lower corner of the scapula. The upper part is covered by the pectoralis major and minor muscles while the interior is covered by the intercostal muscles and parietal pleura. The lateral cutaneous branch of the intercostal nerve can pass through the serratus anterior to reach the subcutaneous area. The intercostal nerve is one of the spinal nerves, consisting of the ventral branches of the thoracic nerve with 11 on each side. The innermost muscles of the wall pleura and intercostals are accompanied by intercostal blood vessels under the ribs. The lateral cutaneous branch is divided near the midaxillary line, passing through the intercostal muscle and the anterior serratus muscle, and is responsible for sensory innervation of the corresponding skin and subcutaneous tissue.

In 2013, Blanco et al. (1) proposed SAPB on the basis of Pectoralis muscles (PECS) blocks I and PECS II. Blanco selected four volunteers in the supine position, and the ultrasound probe was positioned on the fifth rib of the midaxillary line. At this time, the latissimus dorsi and anterior serratus muscle covering the fifth rib were easily recognized using ultrasound. A 22 G needle was inserted into the latissimus dorsi and the serratus anterior (superficial block of serratus anterior) to inject $0.125 \%$ levobupivacaine $4 \mathrm{~mL} / \mathrm{kg}$. It can also be inserted through the serratus anterior and injected under the serratus anterior (deep block of the serratus anterior). After 30 minutes, the acupuncture test was used to determine sensory loss the T2-T9 plane.

\section{Block range and drug selection of $S A P B$}

\section{Blocking range and its influencing factors}

Mayes et al. (2) performed SAPB on six cadavers with the ultrasound guide and found that the sawtooth plane block appeared to be mediated by blocking the lateral cutaneous branch of the intercostal nerve. Kunigo et al. (3) selected nine adult cadavers, each of which was guided by the ultrasound-guided SAPB, injected with $20 \mathrm{~mL}$ methylene blue dye on one side and $40 \mathrm{~mL}$ on the opposite side, and dissected the chest wall after 20 minutes of injection. It was found that the T2-T5 intercostal nerves were completely stained in the group with $40 \mathrm{~mL}$ methylene blue, while only the T3-T4 nerves were completely stained in the group with $20 \mathrm{~mL}$ methylene blue. This experiment shows that the ultrasound-guided SAPB has a wider range of injections in the $40 \mathrm{~mL}$ group than in the $20 \mathrm{~mL}$ group. However, the failure to achieve T2-T9 sensory loss in the Blanco experiment may be due to the gap between clinical effects and anatomical evaluation. Another experimental study suggests that the block range of SAPB is mainly affected by the injection volume of local anesthetics rather than the injection level. The solution diffusion range is similar in both the surface of the anterior serratus muscle and the deep surface of the serratus anterior muscle, and doubling the dose doubles the injection diffusion area and preferentially promotes the diffusion of the dye to the anterior chest wall (4).

\section{Selection of local anesthetics and addition of adjuvants}

According to studies and case reports, the commonly used local anesthetics for clinical SAPB are ropivacaine and bupivacaine. Huang et al. (5) used a randomized doubleblinded experiment to compare the analgesic time and the effect of different concentrations $(0.375 \%, 0.5 \%$, and $0.75 \%)$ of ropivacaine in breast surgeries. The results showed that the higher the ropivacaine concentration, the longer the sensory block time. Compared with $0.5 \%$ ropivacaine and $0.75 \%$ ropivacaine, there was no significant difference in postoperative analgesia, but both were better than $0.375 \%$ ropivacaine. Therefore, $0.5 \%$ ropivacaine block is more suitable for analgesia after mammary gland surgery. Abdallah et al. (6) proposed in a prospective randomized controlled study that the use of levobupivacaine combined with dexmedetomidine in continuous SAPB for analgesia after thoracotomy is a promising option. Compared with levobupivacaine alone, the addition of dexmedetomidine can further reduce the postoperative visual analog scale (VAS) score and the dosage of opioids, and it has good analgesic and sedative effects. In addition, El Sherif et al. (7) added $10 \mathrm{mg}$ of morphine hydrochloride during SAPB after modified radical mastectomy. The results showed that the addition of morphine can reduce the VAS pain scores after rest or exercise and prolong the analgesia time. The addition of adjuvant can block various nociceptive conduction pathways, enhance the analgesic effect of anterior serratus muscle plane block, prolong the block time, and meet the requirements of multimodal 
analgesia.

At present, there are few clinical studies on the injection volume and optimal concentration of local anesthetics. In the future, further research is needed to determine the best dosing regimen to achieve the best analgesia while avoiding potential toxic and side effects.

\section{Clinical application}

$\mathrm{SAPB}$ is considered as thoracic epidural and vertebral because of its simple and easy operation and accurate analgesic effect, which reduces the use of opioids during and after surgery and prevent hemodynamic fluctuations, nerve damage, and other adverse reactions. It is considered an alternative to paraneural blockade (8). Therefore, it is widely used clinically in breast surgery, thoracoscopic surgery, and thoracotomy.

\section{Breast surgery}

Small breast surgery can cause significant postoperative pain. Modified radical mastectomy (MRM) is the common surgical method for breast cancer. It requires the removal of the axillary lymph nodes, the entire breast, and a large amount of skin and subcutaneous tissue. Nearly $60 \%$ of patients undergoing breast surgery will have severe acute pain after the operation, which affects the patient's postoperative recovery (9). A meta-analysis study showed that $\mathrm{SAPB}$ reduced postoperative pain scores more in patients who underwent breast and thoracic surgeries compared with non- block care (10). Moreover, the postoperative analgesic effect of SAPB was superior to that of the paravertebral block (PVB). The SPB was a less invasive alternative to $\mathrm{PVB}$ for providing postoperative analgesia after breast surgery. SAPB does not only inhibits the postoperative acute pain of breast cancer but also treats chronic pain after breast cancer surgery. A clinical report shows an old woman who underwent a left partial mastectomy and axillary node dissection for breast cancer. After surgery, she suffered chronic pain and failed to respond to medication. The operator treated her with SAPB with $10 \mathrm{~mL}$ of $1 \%$ lidocaine, which was repeated 10 times for six months. After that, she has not required any pain management. The serratus plane block better targets the web of nerves innervating the anterior chest wall, including the breast, which is performed more safely and easily than neuraxial approaches (11). Mazzinari et al. (12) used a randomized controlled experiment to compare the analgesic effect of SAPB and conventional intravenous analgesia after breast surgery. Sixty patients who underwent breast cancer surgery were selected and were randomly divided into two groups: conventional analgesia (control group, $\mathrm{n}=30$ ) or single-injection serratus block with L-bupivacaine $0.25 \% 30 \mathrm{~mL}$ (study group, $\mathrm{n}=30$ ). The total morphine consumption 24 hours postoperatively, pain scores 1, 3, 6, 12 , and 24 hours postoperatively, time-to-first opioid rescue analgesia, and the adverse reactions. The results showed that compared with traditional intravenous analgesia, the SAPB group had a lower postoperative pain score, which reduced the need for opioids after breast cancer surgery since the pain was delayed. With the proposition of multimodal analgesic strategies, Wang et al. (13) proposed the combination of thoracic nerve block I and SAPB for postoperative analgesia after MRM. The results showed that the combined application of thoracic nerve block I and SAPB can significantly reduce the use of opioids in the perioperative period and relieve postoperative pain better for breast cancer surgery. This study also provided a new idea for multimodal analgesia strategy.

\section{Thoracoscopic surgery}

Thoracoscopic technique is widely used in thoracic surgery because of its small incision, which can reduce mortality, improve postoperative pulmonary function, and shorten hospital stay (14). Although the incision is small, the postoperative pain is obvious, affecting the expectoration of patients, which can lead to complications, such as pulmonary infection and delayed recovery of patients. Therefore, effective postoperative analgesia can reduce the incidence of pulmonary infection (15). Viti et al. (16) randomly divided 94 patients undergoing Videoassisted thoracoscopic surgery major lung resection into two groups: the control group, which received intravenous and oral (i.e., systemic) analgesics and the intervention group, which received systemic analgesics plus pre-emptive SAPB. They found that SAPB provided better pain control, entailing a better performance during postoperative rehabilitation exercises in terms of duration and quality of incentive spirometry. It diminished the patient's need for rescue analgesics during the early postoperative period. Other studies suggest that SAPB plays an important role in analgesia after thoracoscopic surgery $(17,18)$. SAPB can reduce the amount of morphine needed and the incidence of postoperative vomiting after thoracoscopic surgery, suggesting that SAPB is an effective adjuvant treatment option for postoperative analgesia after thoracic surgery. It can also be seen that the analgesic effect of SAPB and 
TPVB after thoracoscopic surgery is similar (19). SAPB and TPVB are equally effective, simple to operate, and safe for reducing postoperative pain. Chen et al. (20) compared the effects of SAPB and incision infiltration anesthesia on pain after thoracoscopic lobectomy and segmentectomy using a randomized controlled study and found that $0.25 \%$ ropivacaine $0.4 \mathrm{~mL} / \mathrm{kg}$ of SAPB can reduce the use of perioperative opioids, reduce VAS pain score, prolong the time required for the first analgesia, and have better analgesic effect compared with local infiltration anesthesia.

\section{Thoracotomy}

Thoracic surgery is a common treatment of thoracic surgery diseases. Due to its long incision, it often causes significant postoperative pain. A good postoperative analgesic effect can effectively reduce patient's postoperative stress response and promote early recovery (21). Due to complex factors, such as blood coagulation, anticoagulation, and hemodynamic instability, pain following thoracotomy is often difficult to handle in patients with cardiac surgery. Kazior et al. (22) conducted an observational cohort study and selected 20 patients who underwent minimal invasive thoracotomy. Patients who underwent valvular thoracotomy were randomly divided into two groups. One group received SAPB under continuous ultrasound guidance, $30 \mathrm{~mL}$ after entering the intensive care unit and $30 \mathrm{~mL}$ of $0.5 \%$ ropivacaine and $4 \mathrm{mg}$ dexamethasone was injected deep into their serratus anterior muscle. Another blank control group was set up. Compared with the control group, the amount of opioids in the group receiving SAPB significantly reduced within 24 hours after surgery, and the amount of opioids in the entire treatment process also decreased while patients who received SAPB scored higher in motivational spirometry without obstruction-related complications. This experiment proves that SAPB may be safe and effective for postoperative analgesia of minimally invasive valve thoracotomy for patients undergoing cardiac surgery, and it supports the development of larger-scale research. Pain syndrome after thoracotomy (PTPS) refers to pain that persists along the thoracotomy scar at least two months postoperatively, which seriously affects the physical and mental health of patients after surgery. Reyad et al. (23) conducted a randomized controlled study to compare the effect of continuous ultrasound-guided SAPB and patient-controlled analgesia (PCA) on the incidence of PTPS in patients with thoracic tumors after thoracotomy. It was found that the incidence of PTPS in the PCA group was significantly higher than that in the SAPB group 8th and 12th weeks postoperatively, and the PTPS patients in the SAPB group had significantly lower demand for pain treatment than the PCA group. The VAS score in the PCA group was significantly higher than that in the SAPB group during activity or resting within 24 hours after surgery. This study shows that SAPB is a good choice for analgesia after thoracotomy. Continuous SAPB catheterization can reduce the appearance of PTPS and the patient's need for pain treatment. SAPB in cardiothoracic surgery can reduce postoperative pain scores and effectively reduce postoperative opioid use. Continuous SAPB can prolong the block time if the catheter is placed, and its effect may be similar to thoracic epidural analgesia and blood flow dynamically stable (24).

\section{Rib fracture}

Rib fractures are very common in chest trauma. Effective and reasonable analgesia can make patients cough and expectorate early, exercise respiratory function, and reduce the incidence of infection and mortality. Martinez et al. (25) selected 10 patients with blunt chest trauma and rib fractures. Among the 10 patients, three were treated with $\mathrm{SAPB}$, and the rest were treated with SAPB for shortterm analgesia, followed by continuous infusion of $0.2 \%$ ropivacaine at a rate of $0.15 \mathrm{~mL} / \mathrm{kg} / \mathrm{hr}$. The results showed that SAPB can significantly reduce the daily oral morphine use of patients and the Numeric Pain Rating Scale score during a cough. It shows that SAPB is effective in reducing the demand for opioids and improving the pain experience of patients with blunt chest trauma. When the patient cannot move due to severe trauma, SAP is a simple, safe, and effective alternative to the epidural catheter methods. Rose et al. (26) reported a case of 3-10 anterolateral and posterior rib fractures with flail chest. After admission, oral analgesics and hydromorphone and intravenous infusion of ketamine and lidocaine were used for PCA. The patient's Numerical Rating Score (NRS) was still as high as 10/10 when the patient took a deep breath, unable to cough and expectorate, and the lower lobe lung became more atelectatic. SAPB was placed in a tube, and $0.2 \%$ ropivacaine was continuously infused at $5 \mathrm{~mL} / \mathrm{hr}$. After 15 minutes, the patient could cough and breathe deeply. After 48 hours, a reexamination of the chest X-ray showed a reduction of atelectasis. Five days later, the patient was discharged from the hospital with a tube, and the NRS pain score reduced to $2 / 10$. The patient had the catheter removed at home on the 7 th day after surgery, and there was no adverse reaction, such as infection or local anesthetic 
poisoning. The SAPB juxtaposition tube provides effective analgesia, which is essential for reducing the morbidity and mortality of patients with multiple rib fractures. Patients with chest trauma, such as rib fractures, can develop secondary injuries, such as atelectasis, pneumonia, and acute respiratory distress syndrome due to the initial chest trauma, leading to high mortality. Effective analgesia can be achieved by making patients breathe deeply, cough effectively, and promote movement early to reduce the risk of respiratory complications. SAPB is a promising planar block technique in thoracic trauma and rib fracture surgery because it is simple to operate and does not require patients to be in a special position. SAPB also has exact analgesic effect and no relevant contraindications.

\section{Upper abdominal surgery}

Blanco believes that the SAPB block range can reach T2T9, which can provide analgesia for upper abdominal wall incisions. Tao et al. (27) applied SAPB and placed a tube during hepatectomy, and injected $10 \mathrm{~mL} 0.75 \%$ ropivacaine into the deep part of the serratus anterior plane at the midaxillary line at T6 and T7, respectively. The tube was placed and $0.15 \%$ ropivacaine was infused at a rate of $5 \mathrm{~mL} / \mathrm{hr}$. The catheter was extubated 48 hours postoperatively. The results showed that SAPB provides good analgesia, reduces the use of opioids after surgery, prevents complications, such as nausea and vomiting.

Theoretically, complications of SAPB include infection, pneumothorax, hematoma caused by vascular injury, local anesthetic poisoning caused by intravascular injection, and hemodynamic fluctuations. However, a review of the literature shows that few obstructive complications occur. This may be due to the relatively simple operation of SAPB, the superficial position of the anterior serrated muscle space, and the relatively small surrounding vessels. The safety of SAPB can be further improved under ultrasonic localization.

\section{Prospects}

Anterior serratus planar block is a new type of regional block that is simple and effective in analgesia and has no adverse effects, such as respiratory and circulatory depression. Compared with traditional local infiltration anesthesia, SAPB requires less local anesthetics, does not cause local anesthetic poisoning reaction, and prolongs the analgesic time through catheterization. Compared with thoracic epidural block, SAPB does not cause spinal cord injury, epidural hematoma, respiratory depression, and hemodynamic fluctuations (28). Compared with intercostal nerve block, SAPB is simple to conduct, does not need more point injection and has no severe complications, such as pneumothorax. Compared with thoracic paravertebral nerve block, SAPB is less difficult and has no risk of orthostatic hypotension and urinary retention (29). Compared with total intravenous analgesia, SAPB has no adverse reactions, such as nausea and vomiting, excessive sedation, and respiratory depression caused by opioids, and opioids are more expensive. Therefore, we may consider that future research on SAPB can be performed in an ambulatory surgery, such as breast nodule resection, breast prosthesis implantation, invasive operations, such as breast tissue pathological biopsy and treatment of intercostal neuralgia. However, we are concerned about the types of local anesthetics used in SAPB, the onset time, the duration of action, and the best local anesthetics concentration and capacity. We still do not know whether surgical incisions interfere with the plane of the block and the distribution of local anesthetics. Furthermore, the related complications associated with SAPB are not very clear. Further studies are still needed to verify its clinical application.

\section{Conclusions}

In summary, SAPB is a simple and safe regional block technology, and its safety and effectiveness have been greatly improved under the guidance of ultrasound, which is worthy of popularization in clinical practice. Under the guidance of ultrasound, SAPB not only reduces the amount of opioids during the perioperative period but also is easy to operate and has a good analgesic effect, making it an alternative for painless and comfortable medical treatment.

\section{Acknowledgments}

Funding: This work is supported by the National Natural Science Foundation of China (No. 81770295) and the Key Project of Excellent Youth in Higher Education Institution of Anhui Province (gxyqZD2018028).

\section{Footnote}

Reporting Checklist: The authors have completed the Narrative Review reporting checklist. Available at http:// 
dx.doi.org/10.21037/apm-20-1542

Conflicts of Interest: All authors have completed the ICMJE uniform disclosure form (available at http://dx.doi. org/10.21037/apm-20-1542). The authors have no conflicts of interest to declare.

Ethical Statement: The authors are accountable for all aspects of the work in ensuring that questions related to the accuracy or integrity of any part of the work are appropriately investigated and resolved.

Open Access Statement: This is an Open Access article distributed in accordance with the Creative Commons Attribution-NonCommercial-NoDerivs 4.0 International License (CC BY-NC-ND 4.0), which permits the noncommercial replication and distribution of the article with the strict proviso that no changes or edits are made and the original work is properly cited (including links to both the formal publication through the relevant DOI and the license). See: https://creativecommons.org/licenses/by-nc-nd/4.0/.

\section{References}

1. Blanco R, Parras T, McDonnell JG, et al. Serratus plane block: a novel ultrasound-guided thoracic wall nerve block. Anaesthesia 2013;68:1107-13.

2. Mayes J, Davison E, Panahi P, et al. An anatomical evaluation of the serratus anterior plane block. Anaesthesia 2016;71:1064-9.

3. Kunigo T, Murouchi T, Yamamoto S, et al. Injection Volume and Anesthetic Effect in Serratus Plane Block. Reg Anesth Pain Med 2017;42:737-40.

4. Biswas A, Castanov V, Li Z, et al. Serratus Plane Block: A Cadaveric Study to Evaluate Optimal Injectate Spread. Reg Anesth Pain Med 2018;43:854-8.

5. Huang L, Zheng L, Wu B, et al. Effects of Ropivacaine Concentration on Analgesia After Ultrasound-Guided Serratus Anterior Plane Block: A Randomized DoubleBlind Trial. J Pain Res 2020;13:57-64.

6. Abdallah NM, Bakeer AH, Youssef RB, et al. Ultrasoundguided continuous serratus anterior plane block: dexmedetomidine as an adjunctive analgesic with levobupivacaine for post-thoracotomy pain. A prospective randomized controlled study. J Pain Res 2019;12:1425-31.

7. El Sherif FA, Abd El-Rahman AM, Othman AH, et al. Analgesic Effect of Morphine Added to Bupivacaine in Serratus Anterior Plane Block Following Modified Radical
Mastectomy. Only a Local Effect? Randomized Clinical Trial. J Pain Res 2020;13:661-8.

8. Franco CD, Inozemtsev K. Refining a great idea: the consolidation of PECS I, PECS II and serratus blocks into a single thoracic fascial plane block, the SAP block. Reg Anesth Pain Med 2019. [Epub ahead of print].

9. Bakr MA, Mohamed SA, Mohamad MF, et al. Effect of Dexmedetomidine Added to Modified Pectoral Block on Postoperative Pain and Stress Response in Patient Undergoing Modified Radical Mastectomy. Pain Physician 2018;21:E87-96.

10. Chong M, Berbenetz N, Kumar K, et al. The serratus plane block for postoperative analgesia in breast and thoracic surgery: a systematic review and meta-analysis. Reg Anesth Pain Med 2019. [Epub ahead of print].

11. Takimoto K, Nishijima K, Ono M. Serratus Plane Block for Persistent Pain after Partial Mastectomy and Axillary Node Dissection. Pain Physician 2016;19:E481-6.

12. Mazzinari G, Rovira L, Casasempere A, et al. Interfascial block at the serratus muscle plane versus conventional analgesia in breast surgery: a randomized controlled trial. Reg Anesth Pain Med 2019;44:52-8.

13. Wang W, Song W, Yang C, et al. Ultrasound-Guided Pectoral Nerve Block I and Serratus-Intercostal Plane Block Alleviate Postoperative Pain in Patients Undergoing Modified Radical Mastectomy. Pain physician 2019;22:E315-23.

14. Bendixen M, Jørgensen OD, Kronborg C, et al. Postoperative pain and quality of life after lobectomy via video-assisted thoracoscopic surgery or anterolateral thoracotomy for early stage lung cancer: a randomised controlled trial. Lancet Oncol 2016;17:836-44.

15. Hanley C, Wall T, Bukowska I, et al. Ultrasound-guided continuous deep serratus anterior plane block versus continuous thoracic paravertebral block for perioperative analgesia in videoscopic-assisted thoracic surgery. Eur J Pain 2020;24:828-38.

16. Viti A, Bertoglio P, Zamperini M, et al. Serratus plane block for video-assisted thoracoscopic surgery major lung resection: a randomized controlled trial. Interact Cardiovasc Thorac Surg 2020;30:366-72.

17. Semyonov M, Fedorina E, Grinshpun J, et al. Ultrasoundguided serratus anterior plane block for analgesia after thoracic surgery. J Pain Res 2019;12:953-60.

18. Park MH, Kim JA, Ahn HJ, et al. A randomised trial of serratus anterior plane block for analgesia after thoracoscopic surgery. Anaesthesia 2018;73:1260-4.

19. Wang L, Wang Y, Zhang X, et al. Serratus anterior plane 
block or thoracic paravertebral block for postoperative pain treatment after uniportal video-assisted thoracoscopic surgery: a retrospective propensity-matched study. J Pain Res 2019;12:2231-8.

20. Chen G, Li Y, Zhang Y, et al. Effects of serratus anterior plane block for postoperative analgesia after thoracoscopic surgery compared with local anesthetic infiltration: a randomized clinical trial. J Pain Res 2019;12:2411-7.

21. Saad FS, EI Baradie SY, Abdel Aliem MAW, et al. Ultrasound-guided serratus anterior plane block versus thoracic paravertebral block for perioperative analgesia in thoracotomy. Saudi J Anaesth 2018;12:565-70.

22. Kazior MR, King AB, Lopez MG, et al. Serratus anterior plane block for minimally invasive valve surgery thoracotomy pain. J Clin Anesth 2019;56:48-9.

23. Reyad RM, Shaker EH, Ghobrial HZ, et al. The impact of ultrasound-guided continuous serratus anterior plane block versus intravenous patient-controlled analgesia on the incidence and severity of post-thoracotomy pain syndrome: A randomized, controlled study. Eur J Pain 2020;24:159-70.

24. Jack JM, McLellan E, Versyck B, et al. The role of

Cite this article as: Xie C, Ran G, Chen D, Lu Y. A narrative review of ultrasound-guided serratus anterior plane block. Ann Palliat Med 2021;10(1):700-706. doi: 10.21037/apm-20-1542 serratus anterior plane and pectoral nerves blocks in cardiac surgery, thoracic surgery and trauma: a qualitative systematic review. Anaesthesia 2020;75:1372-85.

25. Martinez T, Belveyre T, Lopez A, et al. Serratus Plane Block Is Effective for Pain Control in Patients With Blunt Chest Trauma: A Case Series. Pain Pract 2020;20:197-203.

26. Rose P, Ramlogan R, Madden S, et al. Serratus anterior plane block home catheter for posterior rib fractures and flail chest. Can J Anesth 2019;66:997-8.

27. Tao K, Xu H, Zhu C, et al. Serratus anterior plane block catheter for hepatectomy: A method to decrease opioid use perioperatively. J Clin Anesth 2020;61:109682.

28. Xu YJ, Sun X, Jiang H, et al. Randomized clinical trial of continuous transversus abdominis plane block, epidural or patient-controlled analgesia for patients undergoing laparoscopic colorectal cancer surgery. Br J Surg 2020,107:e133-41.

29. Allain PA, Carella M, Agrafiotis AC, et al. Comparison of several methods for pain management after video-assisted thoracic surgery for pneumothorax: an observational study. BMC Anesthesiol 2019;19:120. 